\section{Author Correction: Rebuilding marine life}

https://doi.org/10.1038/s41586-021-03271-2

Correction to: Nature https://doi.org/10.1038/s41586-020-2146-7

Published online 01 April 2020

Check for updates

Carlos M. Duarte, Susana Agusti, Edward Barbier, Gregory L. Britten, Juan Carlos Castilla, Jean-Pierre Gattuso, Robinson W. Fulweiler, Terry P. Hughes, Nancy Knowlton, Catherine E. Lovelock, Heike K. Lotze, Milica Predragovic, Elvira Poloczanska, Callum Roberts \& Boris Worm

Following publication of this Article, correspondence with colleagues and subsequent analysis uncovered discrepancies between the spreadsheet containing data from the RAM Legacy Stock Assessment Database we used for this analysis and the content of the database, which is now publicly available ${ }^{1}$. In particular, a set of numerical entries for 'spawning stock biomass' and 'total stock biomass' are numerically incorrect or missing in the spreadsheet analysed for the paper. We have been unable to trace back the origin of these differences. As the updated database has now been published online ${ }^{1}$, we wish to ensure reproducibility by correcting the published record to be consistent with the publicly available database.

We have re-run all analyses that use the data in question. This included recalculating: (1) the proportions of stocks in increasing/decreasing/ no change' biomass categories (Fig. 3a and Supplementary Fig. S2.3 of the original Article); (2) the aggregate $B / B_{\mathrm{MSY}}$ time series across all global stocks (Fig. 4a of the original Article); (3) the recovery probabilities for individual stocks currently depleted below $B_{\mathrm{MSY}}$ (Fig. $4 \mathrm{~b}$ of the original Article); and (4) the validation of the $B_{\mathrm{MSY}}$ approximation in cases where assessment-estimated $B_{\mathrm{MSY}}$ values are not available (Supplementary Fig. S2.2 of the original Article). We made a methodological modification to the way the aggregate $B / B_{\mathrm{MSY}}$ was calculated for the original Fig. 4a. We originally weighted each stock by their observed biomass value per year; we now weight by the mean annual biomass calculated across years. The motivation for the original choice was to weight stocks according to their contribution to global biomass. In the corrected database, the time series for Japanese sardine now inappropriately dominates the original calculations. The Japanese sardine is one of the largest stocks in the database. Its stock assessment (as now reflected in the RAM Legacy Stock Assessment Database ${ }^{1}$ ) gives an estimate for $B / B_{\mathrm{MSY}}$ that exceeded 30.0 during the 1980 s and declines to a value of 0.1 in the early 2000 s. This represents an overly influential outlier in the database, absent from the previous dataset, in which the vast majority of stocks vary with $B / B_{\mathrm{MSY}}$ between 0.01 and 2.0 , and very rarely over 3.0 or 4.0. This variation, combined with the large overall size of the stock, make the original weighting untenable because it produces a global aggregate trend that mirrors Japanese sardine through the $1980 \mathrm{~s}$ and is unrepresentative of the global fishery. Weighting by mean annual biomass was the simplest modification. Calculating the weights in this way achieves the intended goal of weighting stocks according to their contribution to global biomass, while removing the extreme dominance of single large stocks. Because the number of affected stocks is small relative to the total number of analysed stocks $(<10 \%)$, other trends remained unchanged, or required small numerical updates.

The changes affect Figs. 3a, 4, Supplementary Figs. S2.2, S2.3 and Supplementary Table S1 of the original Article. Figures 3a, 4, and Supplementary Figs. S2.2 and S2.3 have been redrawn. Figure 1 of this Amendment shows the original and corrected Fig. 3a side by side, for transparency. The proportions of species in the pie chart categories shifted slightly with the corrected database. The data continue to show that the proportion of increasing stocks has improved after year 2000. Figure 2 of this Amendment shows the original and corrected Fig. 4, for transparency. The number of stocks with biomass below the biomass supporting the maximum sustainable yield $\left(B_{\mathrm{MSY}}\right)$ increased from $n=$ 172 in the original paper to $n=194$ using the corrected database. The recovery histograms were re-computed using the updated list of stocks. The updated list of stocks is given in Supplementary Table S1. The legend of Fig. 4 has been updated to reflect the sample size and version number of the corrected database. In the redrawn Fig. 4a, the updated calculations raise the average value of the mean globally aggregated $B / B_{\mathrm{MSY}}$ time series above where it was originally displayed, primarily owing to the treatment of Japanese sardine. These changes do not alter any of our conclusions or the main text of the Article. All text and conclusions are based on the ability to recover depleted individual stocks (Fig. 4b), which does not involve the global aggregate calculations. The recovery of individual stocks is calculated from stock-specific growth rates and fishing pressure, and the probability of recovering all depleted stocks is calculated by combining the stock-specific recovery probabilities.

Supplementary Table S1 also now contains three additional columns, to clarify more precisely the selection criteria; specifically, we added indicators for the availability of total biomass (TB), total catch (TC),
Original, incorrect figure $3 a$



Corrected figure $3 a$

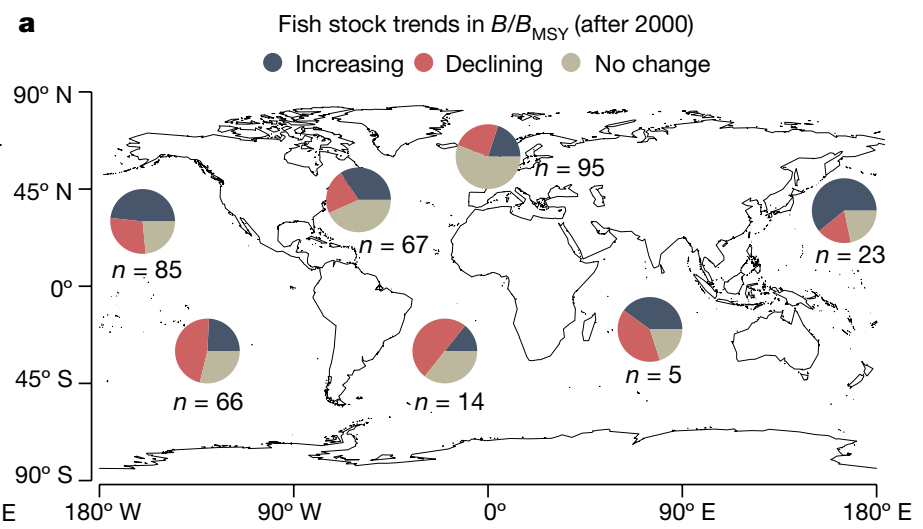

Fig. 1 This figure shows the original and corrected Fig. 3a of the original Article. The original, incorrect Fig. $3 a$ is on the left and the corrected Fig. 3 a is on the right. 
Original, incorrect figure 4
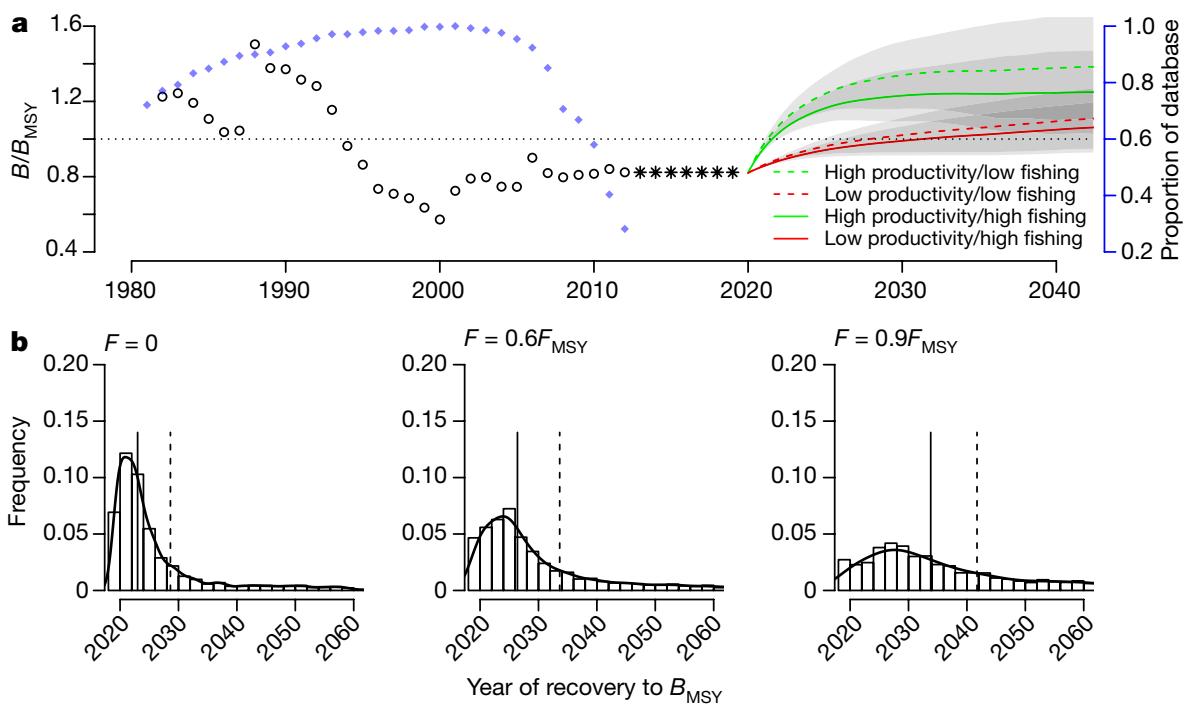

Corrected figure 4
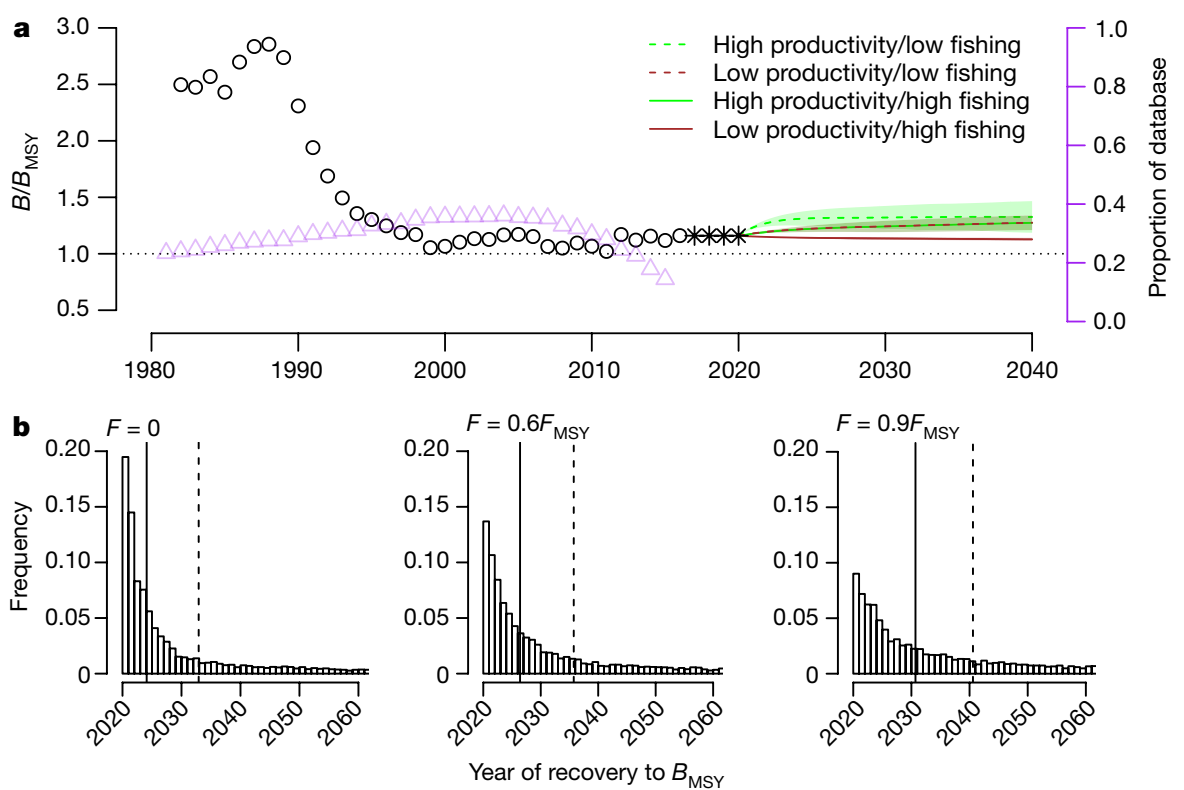

Fig. 2 | This figure shows the original and corrected Fig. 4 of the original Article.

and the most recent estimate of the biomass relative to the target (TB/TB $\left.\mathrm{TBS}_{\mathrm{MS}}\right)$. The simulations continue to show that recovery to 2050 is highly probable and that the probability of recovery increases as fishing pressure decreases. For Supplementary Fig. S2.2, we re-calculated the metrics used to validate our $B_{\mathrm{MSY}}$ approximation for cases where the assessment does not provide an estimate. The validation continues to show that $B_{\mathrm{MSY}}=0.5 \mathrm{max}(B)$ approximates the assessment $B_{\mathrm{MSY}}$ well.

A copy of RAM Legacy 4.491 and the code we used to perform and visualize the $B / B_{\mathrm{MSY}}$ calculations is available at the public GitHub page https://github.com/gregbritten/duarte-etal-correction. The Supplementary Information of this Amendment is the originally published, incorrect Supplementary Information of the original Article, for transparency to our readers. Figures 3 and 4 and the Supplementary Information of the original Article have been corrected online.

Supplementary Information is available in the online version of this Amendment.

1. RAM Legacy Stock Assessment Database version RAMLDB v4.491, http://zenodo.org/ record/3676088 (2020) 